\title{
NOSARA, MALEKE E INTAFRIT NUEVAS VARIEDADES DE PAPA (Solanum tuberosum) PARA COSTA RICA
}

\author{
Nevio A. Bonilla
}

\begin{abstract}
RESUMEN
En el período comprendido entre 1993 y 2003 se evaluaron una serie de clones de papa con el objeto de determinar genotipos superiores que pudieran ser considerados como variedades comerciales. Se realizaron ensayos de introducción, evaluación preliminar, rendimiento, verificación y validación de colecciones procedentes del Centro Internacional de la Papa (CIP) y el programa de mejoramiento genético del INIFAP de México. Se evaluaron características de adaptación, potencial de rendimiento, calidad física y las propiedades sensoriales de dichos clones tanto en pruebas experimentales como semicomerciales. Este trabajo se llevó a cabo tanto en la Estación Experimental Carlos Durán como en fincas de productores de la zona norte de Cartago y de Zarcero. Se determinó que los clones denominados 391683.80 (NOSARA), 387096.11 (MALEKE) y 387146.47 (INTAFRIT) presentan características de rendimiento, adaptación y calidad de producto adecuadas para ser considerados como nuevas variedades de papa. Durante el proceso de evaluación mostraron un rendimiento superior a $35 \mathrm{t} / \mathrm{ha}$, tolerancia a tizón tardío y buenas propiedades para consumo fresco, en el caso de NOSARA y MALEKE, así como para hojuelas en el caso de INTAFRIT con valores superiores a $20 \%$ de materia seca.
\end{abstract}

Palabras clave: Papa (solanum tuberosum), mejoramiento, selección, adaptación, rendimientos.

\section{INTRODUCCIÓN}

La papa es una de las hortalizas más importantes de nuestro país, tanto por la actividad económica asociada a su producción, como por el aporte de vitaminas y carbohidratos a la dieta nacional (Beukema 1990; FAO 1994; Horton 1992; Vázquez 1988). Los procesos de globalización y apertura comercial exigen que las actividades productivas en el campo agrícola incorporen de manera efectiva la competitividad como elemento indispensable para poder continuar siendo alternativas válidas en la economía del país. En este sentido, el cultivo de la papa requiere de alternativas tecnológicas como nuevas variedades con características adecuadas para producción, mercadeo y consumo.

En Costa Rica se siembran anualmente alrededor de 3.000 ha de papa, principalmente en la zona norte de Cartago, Zarcero, alrededores del Cerro de la Muerte y más recientemente en la cordillera volcánica norte
(Guanacaste), donde se ubican aproximadamente 1.000 productores de papa comercial y de semilla. Para el año 1999 se sembraron 2.802 ha, se produjeron 66.700 toneladas métricas y el rendimiento promedio fue de 23,8 t /ha (CNP 2000). La industria de papa en los últimos años ha asumido un papel muy importante en el impulso de la demanda de este producto, siendo ésta de $15-20 \%$ del volumen total anual (Barboza 2000) ${ }^{2}$. Además este sector se ha vuelto más exigente en cuanto a la calidad de la papa procesada y del producto terminado. Se estima también un consumo de 6.000 toneladas métricas anuales por parte de la industria (Barboza 2000). De acuerdo con estudios realizados en las zonas de producción de papa, el $70 \%$ de los productores siembran menos de 3 ha, produciendo globalmente del 55 a $60 \%$ de la producción total y la diferencia o sea el 40-45 $\%$ es producida por el restante $30 \%$ de los productores. Las importaciones mensuales de papa de mesa hasta el mes de abril del 2000

\footnotetext{
${ }^{1}$ Instituto Nacional de Innovación y Transferencia en Tecnología Agropecuaria (INTA), Costa Rica.

${ }^{2}$ Barboza, 2000. Datos sobre la producción de papa. Instituto Tecnológico. Cartago, Costa Rica. Comunicación personal.
} 
ascendieron a 3.704 toneladas métricas, las de papa frita congelada en el año 1999 registraron 14.234 toneladas métricas con un valor estimado por kilogramo de 0,807 dólares. El costo de producción por hectárea fluctúa entre $\$ 3.000,00$ y $\$ 4.000,00$.

El objetivo de este trabajo fue desarrollar nuevas variedades de papa que presenten características de resistencia a las plagas y enfermedades de importancia económica y calidad para la industria de procesamiento (papa frita y tostadas).

\section{MATERIALES Y MÉTODOS}

El presente trabajo se llevó a cabo durante los años 1999 a 2003 en la Región de Cartago (Alvarado, Tierra Blanca, Oreamuno y Llano Grande) y de Alajuela (Zarcero). Se realizaron repeticiones en cada localidad. A continuación se detallan por año y localidad los ensayos de campo (con y sin diseño estadístico) en los que se evaluaron las nuevas variedades de papa. Es importante indicar que los códigos de las nuevas variedades corresponden de la siguiente manera: 391683.80 NOSARA, 387096.11 MALEKE, 387146.47 INTAFRIT

La metodología de evaluación para cada año fue la siguiente:

\section{Año 1993}

Una localidad en la zona norte de Cartago: Oreamuno.

Clon: 387096.11(10 tubérculos).

10 parcelas de un surco de $2 \mathrm{~m}$ de largo separadas $0,80 \mathrm{~m}$ y $0,25 \mathrm{~m}$ entre plantas.

Sin repeticiones. Testigo: Atzimba.

\section{Año 1994}

Una localidad de la zona norte de Cartago: Oreamuno.

Clon: 384321.15 (10 tubérculos).

10 parcelas de un surco de $2 \mathrm{~m}$ largo separadas $0,80 \mathrm{~m}$ y $0,25 \mathrm{~m}$ entre plantas. Sin repeticiones. Testigo: Atzimba.

\section{Año 1995}

Una localidad de la zona norte de Cartago: Oreamuno. Clon:3847096.11, 391683.80 (50 tubérculos).

10 parcelas de dos surcos de $5 \mathrm{~m}$ largo separadas $0,80 \mathrm{~m}$ y $0,25 \mathrm{~m}$ entre plantas. Sin repeticiones. Testigo: Atzimba.

\section{Año 1996}

Dos localidades de la zona norte de Cartago: Oreamuno, Alvarado.

Clon: 387146.47, 391683.80, 384321.15, 387096.11 .

20 parcelas de cinco surcos de $5 \mathrm{~m}$ largo separadas $0,90 \mathrm{~m}$ y $0,25 \mathrm{~m}$ entre plantas. Sin repeticiones. Testigos: Atzimba, Floresta.

Cinco Parcelas de multiplicación de cada material $100-250 \mathrm{~m}^{2}$

\section{Año 1997}

Dos localidades de la zona norte de Cartago: Oreamuno, Alvarado.

Clon: 387146.47, 391683.80, 387096.11, 387052.36, 389666.17.

Cinco parcelas de cinco surcos de $5 \mathrm{~m}$ de largo separadas $0,90 \mathrm{~m}$ y $0,25 \mathrm{~m}$ entre plantas. Sin repeticiones. Testigos: Floresta, Birris, Idiap 92. Parcela de multiplicación de cada material $1.000 \mathrm{~m}^{2}$.

\section{Año 1998}

Dos localidades de la zona norte de Cartago: Oreamuno, Alvarado.

Clon: 384321.15, 391683.80, 387096.11, 389666.17

Cinco Parcelas de cinco surcos de $5 \mathrm{~m}$ de largo separadas $0,90 \mathrm{~m}$ y $0,25 \mathrm{~m}$ entre plantas. Sin repeticiones. Testigos: Floresta, Birris, Idiap 92, Atzimba.

Parcela de multiplicación de cada material $1.000 \mathrm{~m}^{2}$.

Evaluación de clones avanzados de papa. CLONES DE C.I.P. Y CLONES MEXICANOS 1996-1997

387031.8; 386042.3; 389666.17; 387028.10;

387096.11; 391684.26; 391684.115 ; 391685.5; 391685.37; 382150.6; 391685.122; 391683.3; 391684.96; 676026; 7764192; 676014. 
Diseño experimental: No se utilizó diseño por no contarse con la suficiente cantidad de material. Se sembró en tres localidades de Cartago. Se utilizaron 16 clones provenientes de colecciones de C.I.P. y México de 1996 y 1997.

Descripción de la unidad experimental: La parcela constó de dos surcos de $5 \mathrm{~m}$ de largo distanciados $0,90 \mathrm{~m}$ entre sí y con una distancia de 0,20 m entre plantas.

\section{Año 2000}

Tres localidades de la zona norte de Cartago: Oreamuno, Cartago y Alvarado.

Siete clones: 387031.8; 384321.15; 386042.3; 391683.80; 389666.17; 387146.47; 387028.10 (200 tubérculos).

Parcelas de cinco surcos de $8 \mathrm{~m}$ largo separadas $0,80 \mathrm{~m}$.

Sin repeticiones. Testigo: Floresta.

Clones proceden de ensayos regionales.

\section{Año 2001}

Ocho localidades de la zona norte de Cartago y dos localidades de Zarcero.

Dos clones: 391683.80 y 397146.47 .

Dos clones adicionales no todos ambientes: 384321.15 y 387096.11 .

Época seca: Noviembre-Marzo.

Época lluviosa: Mayo-Octubre.

\section{Año 2002}

Ocho localidades de la zona norte de Cartago y dos localidades de Zarcero.

Dos clones: 391683.80 y 397146.47.

Dos clones adicionales no todos ambientes: 384321.15 y 387096.11 .

Los materiales se evaluaron en las dos épocas de siembra más importantes siendo estas: Época seca: Noviembre-Marzo, Época lluviosa: Mayo-Octubre.

Ensayo uniforme de clones avanzados y variedades de papa.

Clon o variedad: IDIAP 92, FLORESTA, FRIPAPA, BIRRIS, IDIAFRIT, MALINCHE, MONTSERRAT, NORTEÑA, TOLLOCAN,
ATLANTIC, 391683.80, 388058.7, 381397.32, 391580.30, 387146.47, 78-199-33, 77-18213, 77-18-335, 77-70-91.

Diseño experimental: No se utilizó diseño por no contarse con la suficiente cantidad de material. Se sembraron en tres localidades de Cartago. Se utilizaron 16 clones provenientes de colecciones del C.I.P. y México de 1996 y 1997.

Descripción de la unidad experimental: La parcela constó de dos surcos de $5 \mathrm{~m}$ de largo distanciados $0,90 \mathrm{~m}$ entre sí y con una distancia de 0,20 m entre plantas.

\section{Ensayo de clones promisorios de papa.}

Clon: 393438.107, 393427.203, 393385.7, $391061.144, \quad 393427.28, \quad 393295.173$, $393385.257, \quad 391046.90, \quad 392631.107$, $393427.199, \quad 393427.3, \quad 392637.269$, 392637.10.

Diseño experimental: No se utilizó diseño por no contarse con la suficiente cantidad de material. Se sembraron en tres localidades de Cartago. Se utilizaron 16 clones provenientes de colecciones del C.I.P. y México de 1996 y 1997.

Descripción de la unidad experimental: La parcela constó de dos surcos de $5 \mathrm{~m}$ de largo distanciados $0,90 \mathrm{~m}$ entre sí y con una distancia de 0,20 m entre plantas.

\section{Ensayo de clones promisorios y variedades de papa.}

Clon o Variedad: 77, 386042.3, 3916783.80, 5750.49, 387096.11, 387031.8, 384321.15, 387146.47, IDIAP, GRANOLA, BIRRIS, FLORESTA, IDIAFRIT.

Diseño experimental: No se utilizó diseño por no contarse con la suficiente cantidad de material. Se sembraron en tres localidades de Cartago. Se utilizaron 16 clones provenientes de colecciones del C.I.P. y México de 1996 y 1997. 
Descripción de la unidad experimental: La parcela constó de dos surcos de $5 \mathrm{~m}$ de largo distanciados $0,90 \mathrm{~m}$ entre sí y con una distancia de 0,20 m entre plantas.

\section{Evaluación de materiales promisorios. Año 2002.}

Durante el período 2002-2003 se evaluaron y validaron los materiales disponibles, enviados en años anteriores por México, Cuba y del C.I.P.. Se establecieron tres ensayos de evaluación de materiales promisorios. Así mismo algunos de ellos fueron seleccionados para su posterior multiplicación, ya que presentaron características adecuadas para ser considerados en futuras liberaciones de materiales.

En este sentido se evaluaron 14 clones provenientes de CIP, 17 clones provenientes del programa de México y una variedad de Cuba. De igual manera se validaron cuatro clones provenientes de CIP de los cuales se seleccionaron dos clones que fueron liberados en abril del 2003. En total se establecieron 10 parcelas de validación de estos dos clones en los diferentes ambientes de las zonas productoras de papa. Estos clones se multiplicaron en invernadero y campo con la colaboración de agricultores para tener cantidades razonables de semilla para su liberación. Dichos clones corresponden a los códigos 391683.80, $387146.47,387096.11$ y 384321.15.

En cuanto a la evaluación de clones promisorios, se establecieron tres ensayos que contemplaron de manera independiente los 14 clones del CIP (un ensayo), los 17 clones de México (un ensayo) y la variedad Marinca de Cuba (incluida en dos ensayos). También se aprovechó para multiplicar estos materiales para su evaluación en fincas de agricultores y en parcelas más grandes con diseño estadístico.

\section{Evaluación de materiales avanzados. Año 2002.}

Dicho ensayo consistió de 19 variedades y clones que se establecieron en una parcela experimental de tres surcos por variedad, con 10 plantas cada uno, a una distancia entre plantas de 0,30 m y 0,80 m entre surcos. La distribución de los materiales en la parcela fue al azar. El ensayo fue establecido en la Estación Experimental Carlos Durán ubicada a $2.350 \mathrm{msnm}$, en el distrito Potrero Cerrado, cantón de Oreamuno, en la provincia de Cartago. El manejo agronómico fue el que comúnmente se realiza en Costa Rica. Se hicieron aplicaciones de fungicidas para el control del Tizón Tardío, debido a la fuerte presión de la enfermedad, y fue necesario aplicar fungicidas preventivos cada 10 días. Los materiales evaluados fueron:

$\begin{array}{lcr}381381.13 & \text { (IDIAP-92); } & 386056.7 \\ \text { (FLORESTA); } & 388790.24 & \text { (FRIPAPA); } \\ 386040.9 \text { (BIRRIS); 381390.30 } & \text { (IDIAFRIT); } \\ \text { MALINCHE; } \quad \text { MONTSERRAT; } & \text { NORTENAA; } \\ \text { TOLLOCAN; } \quad \text { ATLANTIC; } & 391683.80 ; \\ \text { 388058.7; 381397.32; 391580.30; 387146.47; } & \\ \text { 78-199-33; 77-18-335; 77-70-91 } & \end{array}$

\section{Año 2003}

Consistió de un ensayo de rendimiento, para verificar el comportamiento de los clones de interés, en conjunto con las variedades más utilizadas en las zonas de cultivo.

Clon o Variedad: Floresta, 387146.47, 387096.11, 575049, Idiap 92, 386042.3, 384321.15, Idiafrit, 391683.80, Granola.

Diseño experimental: Bloques Completos al azar con cuatro repeticiones.

Descripción de la unidad experimental: La parcela constó de cuatro surcos de $5 \mathrm{~m}$ de largo distanciados $0,90 \mathrm{~m}$ entre sí y con una distancia de 0,20 m entre plantas.

\section{VARIABLES Y MÉTODOS DE EVALUACIÓN}

Comportamiento agronómico

1. Emergencia de plantas 22 días después de la siembra.

2. Días a floración (50 \% plantas con flor). 
3. Período de floración (altura plantas).

4. Días a la cosecha (madurez fisiológica).

\section{Problemas de plagas y enfermedades}

\section{Daño por Liriomyza huidobrensis (escala 1} a 4). *

2. Daño por polillas de la papa (escala 1 a 4). *

3. Porcentaje de severidad de tizón tardío (escala 1 a 9$)$. **

${ }^{*}$ Rodríguez 1988. ** Henfling 1987 Hooker 1981; Landeo 2000; Mendoza et al. 2000; Umareus et al. 1994.

\section{Rendimiento}

1. Rendimiento total, comercial y por categorías.

2. Color de piel y pulpa.

3. Profundidad de yemas ("ojos").

4. Disposición espacial de la producción.

5. Contenido sólidos solubles.

6. Contenido de azúcares reductores.

Forma de analizar los datos: Debido a que no en todos los ensayos se contó con un diseño estadístico en los ensayos enunciados anteriormente, se procedió a tomar datos para las variables mencionadas de acuerdo con el siguiente criterio de evaluación. Es importante indicar que en los ensayos de rendimiento de 2003 se utilizó diseño estadístico con repeticiones.

Criterio de evaluación: Este criterio se constituye en un orden de prioridad para la selección de clones de la siguiente manera:

1. Tizón Tardío: Se eliminaron todos aquellos clones que superen un valor en la escala internacional del C.I.P (1-9).*

2. Rendimiento potencial: Se eliminaron todos aquellos clones que no superaron un valor de 500 gramos por planta o 20 t/ha.

3. Calidad: Se consideraron las características físicas (color de piel y pulpa, profundidad de ojos, forma del tubérculo) y las características químicas (\% sólidos solu- bles, \% azúcares reductores) y se eliminaron aquellos clones que no cumplieron con las normas que el mercado fresco o industrial exigen al respecto para comercializar un tubérculo de papa.

4. Tolerancia a plagas: Se eliminaron todos aquellos clones que no presentaron un grado de tolerancia aceptable a la mosca minadora y polillas de la papa. Esto quiere decir que aunque la planta sea atacada por la plaga, ésta tolera el daño sin que se dé un efecto económico de reducción en el rendimiento (daño no afecte el rendimiento y calidad comercial en valores superiores al $15 \%$ ).

\section{RESULTADOS Y DISCUSIÓN}

En el período comprendido entre los años 1993 y 1999 se evaluaron las materiales de papa en sus etapas iniciales donde se caracterizaron por su potencial de producción y características de calidad como el contenido de sólidos, además se valoró su comportamiento bajo las condiciones agro ecológicas de las principales zonas paperas de Costa Rica (Avilés y Bolaños 1999; Bonilla 1998; Bonilla 2001 b). En el Cuadro 1 se presentan de manera resumida los resultados más importantes de los clones evaluados comparados con dos variedades comerciales testigo.

\section{Año 2000}

A partir del año 2000 se establecieron ensayos de rendimiento con y sin diseño en fincas de productores de papa, además se realizaron ensayos de referencia en la Estación Experimental Carlos Durán. Los resultados obtenidos con estos ensayos fueron los siguientes:

\section{Características químicas y de almace- namiento. Año 2000.}

En contenido de materia seca los clones $387146.47, \quad 391683.80$ y 384321.15 presentaron respectivamente $24 \%, 20 \%$ y 19 $\%$. Se determinó que el clon 384321.15 pudre y se brota en almacenamiento en frío y en 
condiciones normales, mientras que los clones 391683.80 y 387146.47 soportan bien en estas dos condiciones.

\section{Año 2001}

Los mayores rendimientos fueron del clon 382143.15, sin embargo, su comportamiento poscosecha fue inadecuado ya que se pudre y brota en almacén en pocas semanas.

Los clones 391683.80 y 387146.47 mostraron rendimientos intermedios pero sin pudrición y brotación en almacén. Se seleccionaron los clones 391683.80 y 387146.47 para la reproducción de semilla y su posterior liberación como variedades. El clon 382143.15 se evaluará durante un ciclo más para confirmar sus características.

Dos épocas por localidad (promedio de
seca y lluviosa). Estación Experimental Carlos Durán: 31,5 t /ha 387146.47; 22,8 t /ha 391683.80; 36,4 t /ha 384321.15; 32,8 t /ha 387096.11.

CUADRO 1. Resumen de características de rendimiento, calidad y fitosanidad de los clones evaluados durante el período 1993-1998.

\begin{tabular}{|c|c|c|c|c|c|c|}
\hline $\begin{array}{l}\text { Clon ó } \\
\text { Variedad }\end{array}$ & 387096.11 & 387146.47 & 391683.80 & 384321.15 & Floresta & Atzimba \\
\hline $\begin{array}{l}\text { Año } \\
1993\end{array}$ & $\begin{array}{l}\text { PPI: } 10 \% \\
\text { Rendimiento: } \\
9,50 \mathrm{Kg} \\
21 \% \text { Sólidos }\end{array}$ & & & & & \\
\hline 1994 & & & & $\begin{array}{l}\text { Rendimiento } \\
11,88 \mathrm{Kg}\end{array}$ & & \\
\hline 1995 & $\begin{array}{l}\text { Rendimiento: } \\
\text { 0,882 kg/planta } \\
\text { Piel crema } \\
\text { Forma redonda } \\
20 \% \text { Sólidos }\end{array}$ & & $\begin{array}{l}\text { GPI: } 8,7 \% \\
\text { Rendimiento: } \\
0,75 \mathrm{~kg} / \text { planta } \\
\text { Piel rosada } \\
\text { Forma redonda }\end{array}$ & & & \\
\hline 1996 & $\begin{array}{l}\text { PPI: } 1,82 \% \\
\text { Rendimiento: } \\
414 \mathrm{Kg}\left(250 \mathrm{~m}^{2}\right) \\
1,66 \mathrm{~kg} / \mathrm{m}^{2} \\
19,9 \% \text { sólidos }\end{array}$ & $\begin{array}{l}\text { PPI: } 2,55 \% \\
\text { Rendimiento: } \\
161 \mathrm{Kg} / 100 \mathrm{~m}^{2} \\
22,5 \% \text { Sólidos }\end{array}$ & $\begin{array}{l}\text { PPI: } 7,9 \% \\
\text { Rendimiento: } \\
46,5 \mathrm{Kg} \\
\left(50 \mathrm{~m}^{2}\right)\end{array}$ & $\begin{array}{l}\text { PPI: } 3 \\
\text { Rendimiento: } \\
1,25 \mathrm{~kg} / \mathrm{m}^{2}\end{array}$ & 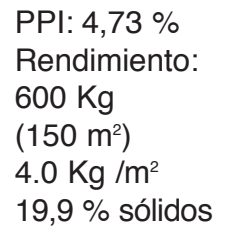 & $\begin{array}{l}\text { PPI: } 12,7 \% \\
\text { Rendimiento: } \\
300 \mathrm{Kg} \\
(100 \mathrm{~m} 2) \\
3,0 \mathrm{~kg} / \mathrm{m} 2 \\
18,0 \% \text { sólidos }\end{array}$ \\
\hline 1997 & $\begin{array}{l}\text { AUDPC: } \\
\text { Rendimiento: } \\
1.700 \mathrm{Kg} \\
\left(1.000 \mathrm{~m}^{2}\right)\end{array}$ & $\begin{array}{l}\text { AUDPC: } \\
\text { Rendimiento: } \\
454 \mathrm{Kg} \\
\left(1.000 \mathrm{~m}^{2}\right)\end{array}$ & $\begin{array}{l}\text { AUDPC: } \\
\text { Rendimiento: } \\
243 \mathrm{~kg} \\
\left(100 \mathrm{~m}^{2}\right)\end{array}$ & AUDPC: & AUDPC:1.780 & \\
\hline 1998 & $\begin{array}{l}\text { AUDPC: } \\
1.202,6 \\
249,12\end{array}$ & $\begin{array}{l}\text { AUDPC: } \\
1.039,2 \\
226.75\end{array}$ & $\begin{array}{l}\text { AUDPC: } \\
1.335 \\
237,5\end{array}$ & $\begin{array}{l}\text { AUDPC: } \\
1.084 .9 \\
154,5\end{array}$ & $\begin{array}{l}\text { AUDPC: } \\
24,75 \\
1.610,75\end{array}$ & $\begin{array}{l}\text { AUDPC: } \\
1.537,5 \\
271,37\end{array}$ \\
\hline
\end{tabular}

PPI: Porcentaje de plantas infectadas.

GPI: Grado Promedio de Infección.

AUDPC: Área bajo la curva de progreso de la enfermedad. 
Bonilla: Nosara, Maleke e Intafrit nuenas variedades de papa (Solanum tuberosum) para Costa Rica.

CUADRO 2. Rendimiento de parcelas de observación de clones. Cartago, 2000.

\begin{tabular}{|c|c|c|c|c|c|c|}
\hline \multirow{2}{*}{$\frac{\text { Localidad }}{\text { Clon }}$} & \multicolumn{2}{|c|}{ Alejo Granados-Oreamuno } & \multicolumn{2}{|c|}{ Wagner Guillén-Alvarado } & \multicolumn{2}{|c|}{ Rosario Brenes-Tierra Blanca } \\
\hline & $\begin{array}{l}\text { Peso (kg/ } \\
\text { parcela) }\end{array}$ & $\begin{array}{l}\text { Rendimiento } \\
\text { comercial } \\
\text { (t/ha) }\end{array}$ & $\begin{array}{l}\text { Peso (kg } \\
\text { /parcela) }\end{array}$ & $\begin{array}{l}\text { Rendimiento } \\
\text { comercial } \\
\text { (t/ha) }\end{array}$ & $\begin{array}{c}\text { Peso (Kg/ } \\
\text { parcela) }\end{array}$ & $\begin{array}{l}\text { Rendimiento } \\
\text { comercial } \\
(\mathrm{t} / \mathrm{ha})\end{array}$ \\
\hline 387031.8 & 118,9 & 33,0 & 35,0 & 19,4 & 122,4 & 34,0 \\
\hline 384321.15 & 127,6 & 35,4 & 120,0 & 33,3 & 72,0 & 20,0 \\
\hline 386042.3 & 73,7 & 20,5 & 22,0 & 12,2 & 86,4 & 24,0 \\
\hline 391683.80 & 122,1 & 33,9 & 32,0 & 17,8 & 104,4 & 29,0 \\
\hline 389666.17 & 108,3 & 30,1 & 93,0 & 25,8 & 169,2 & 47,0 \\
\hline 387146.47 & 98,1 & 27,2 & 52,0 & 28,9 & 104,4 & 29,0 \\
\hline 387028.10 & 116,5 & 32,4 & 40,0 & 22,2 & 108,0 & 30,0 \\
\hline
\end{tabular}

CUADRO 3. Características físicas del tubérculo. Cartago, 2000.

\begin{tabular}{ccccc}
\hline Clon & Forma tubérculo & Color de piel & Color de la pulpa & Profundidad de ojos \\
\hline 387031.8 & Alargada-Ovalada & Crema & Crema & Superficial \\
384321.15 & Alargada & Crema & Blanca & Superficial \\
386042.3 & Ovalada-alargada & Amarilla & Blanca & Superficial \\
391683.80 & Redonda & Rosada & Amarilla & Superficial \\
389666.17 & Alargada-Ovalada & Crema & Blanca & Semiprofundo \\
387146.47 & Redonda & Amarilla & Crema & Semiprofundo \\
387028.10 & Ovalada & Amarilla & Blanca & Profundo \\
\hline
\end{tabular}

Zarcero: $31 \mathrm{t} / \mathrm{ha} 387146.47 ; 35,5 \mathrm{t}$ /ha 391683.80.

Tierra Blanca: 29 t /ha 391683.80; 52,2 t /ha 387146.47.

Llano Grande: $30 \mathrm{t} / \mathrm{ha} 391683.80 ; 45 \mathrm{t} / \mathrm{ha}$ 387146.47.

Pacayas:32 t /ha 391683.80; 43 t /ha 387146.47.

\section{Año 2002}

Los Cuadros 4, 5 y 6 resumen los resultados de estos ensayos y en ellos se indican las características de rendimiento y calidad de tubérculo (físicas y sensoriales). Es importante indicar que las evaluaciones de las variedades NOSARA, INTAFRIT Y MALEKE destacaron por sus características adecuadas de alto rendimiento y buenas cualidades para su consumo fresco, así como para uso industrial en el caso de INTAFRIT. 
ALCANCES TECNOLÓGICOS, AÑO 4, NÚMERO 1

CUADRO 4. Rendimiento de 19 clones y variedades de papa evaluadas en ensayo regional uniforme. Año 2002.

\begin{tabular}{|c|c|c|c|c|c|c|}
\hline \multirow{2}{*}{$\begin{array}{l}\text { Clon o } \\
\text { variedad }\end{array}$} & \multicolumn{6}{|c|}{ Rendimiento kg/parcela* por categoría.** } \\
\hline & $1^{\mathrm{a}}$ & $2^{\mathrm{a}}$ & $3^{\mathrm{a}}$ & $4^{\mathrm{a}}$ & Comercial & No comercial \\
\hline IDIAP-92 & 19,00 & 3,50 & 0,80 & 0,70 & 23,30 & 0,70 \\
\hline FLORESTA & 9,45 & 1,50 & 1,30 & 0,30 & 12,25 & 0,30 \\
\hline FRIPAPA & 3,00 & 3,50 & 2,50 & 1,00 & 9,00 & 1,00 \\
\hline BIRRIS & 21,00 & 1,80 & 0,50 & 0,90 & 23,30 & 1,07 \\
\hline IDIAFRIT & 8,70 & 2,50 & 0,75 & 0,90 & 11,95 & 0,90 \\
\hline MALINCHE & 20,60 & 4,40 & 1,05 & 2,35 & 26,05 & 2,50 \\
\hline NORTEÑA & 10,10 & 3,20 & 0,50 & 0,70 & 13,80 & 0,70 \\
\hline TOLLOCAN & 14,00 & 2,40 & 0,70 & 0,20 & 17,10 & 0,20 \\
\hline ATLANTIC & 4,50 & 1,10 & 0,20 & 0,15 & 5,80 & 0,15 \\
\hline 391683.80 & 12,50 & 1,90 & 0,75 & 0,60 & 15,15 & 0,60 \\
\hline 387096.11 & 13.45 & 1.45 & 0.30 & 0.15 & 15,90 & 0.45 \\
\hline 388058.7 & 18,00 & 2,20 & 0,70 & 0,20 & 20,90 & 0,20 \\
\hline 381397.32 & 9,50 & 1,20 & 0,40 & 0,30 & 11,10 & 0,30 \\
\hline 391580.30 & 6,40 & 1,30 & 0,20 & 0,50 & 7,90 & 0,50 \\
\hline 387146.47 & 19,00 & 2,50 & 0,95 & 0,80 & 22,45 & 0,80 \\
\hline $78-199-33$ & 12,00 & 1,60 & 0,50 & 0,25 & 14,10 & 0,25 \\
\hline $77-18-213$ & 6,20 & 0,80 & 0,50 & 0,10 & 7,50 & 0,10 \\
\hline $77-18-335$ & 16,00 & 1,30 & 0,35 & 0,50 & 17,65 & 1,30 \\
\hline $77-70-91$ & 12,50 & 2,20 & 0,50 & 0,35 & 15,20 & 0,80 \\
\hline
\end{tabular}

* Parcela útil constó de 20 plantas. Las calidades señaladas corresponden a las categorías en que se clasifican comercialmente los tubérculos para la comercialización, tomando en consideración el tamaño, sanidad y daño físico que presenten los tubérculos.

${ }^{* \star}$ Categorías comerciales : $1^{\text {a }}$ : grande; $2^{\mathrm{a}}$ : mediana; $3^{\mathrm{a}}$ :pequeña; $4^{\mathrm{a}}$ :arreflis 
Bonilla: Nosara, Maleke e Intafrit nuenas variedades de papa (Solanum tuberosum) para Costa Rica.

CUADRO 5. Características físicas del tubérculo y contenido de sólidos de 19 variedades y clones de papa evaluadas en ensayo regional. Año 2002.

\begin{tabular}{|c|c|c|c|c|c|}
\hline $\begin{array}{l}\text { Clon o } \\
\text { variedad }\end{array}$ & Forma & $\begin{array}{l}\text { Color } \\
\text { cutícula }\end{array}$ & $\begin{array}{l}\text { Color } \\
\text { pulpa }\end{array}$ & $\begin{array}{l}\text { Profundidad } \\
\text { de ojos }\end{array}$ & $\begin{array}{l}\text { Gravedad } \\
\text { específica* }\end{array}$ \\
\hline IDIAP-92 & Ovalada & Crema & Crema & Superficial & 1,064 \\
\hline FLORESTA & Ovalada & Crema & Blanca & Superficial & 1,063 \\
\hline FRIPAPA & Redonda - Ovalada & Roja & Blanca & Superficial & 1,066 \\
\hline BIRRIS & Ovalada - Aplastada & Blanca & Amarilla & Superficial & 1,060 \\
\hline IDIAFRIT & Alargada- Aplastada & Crema & Blanca & Superficial & 1,060 \\
\hline MALINCHE & Ovalada & Crema & Blanca & Superficial & 1,060 \\
\hline NORTEÑA & Ovalada & Crema & Amarilla & Semiprofundo & 1,065 \\
\hline Tollocan & Alargada & Crema & Blanca & Superficial & 1,062 \\
\hline ATLANTIC & Ovalada- Aplastada & Blanca & Amarilla & Superficial & 1,079 \\
\hline 391683.80 & Redonda & Rosada & Amarilla & Superficial & 1,075 \\
\hline 388058.7 & Ovalado & Crema & Blanca & Superficial & 1,060 \\
\hline 381397.32 & Alargada-Aplastada & Blanca & Blanca & Semiprofundo & 1,062 \\
\hline 391580.30 & Ovalada & Crema & Amarilla & Superficial & 1,064 \\
\hline 387146.47 & Ovalada & Crema & Crema & Superficial & 1,062 \\
\hline $78-199-33$ & Ovalada-Aplastada & Amarilla & Amarilla & Superficial & 1,071 \\
\hline $77-18-213$ & Ovalada & Crema & Amarilla & Semiprofundo & 1,060 \\
\hline 77-18-335 & Ovalada-Aplastada & Crema & Blanca & Superficial & 1,062 \\
\hline $77-70-91$ & Ovalada & Crema & Crema & Superficial & 1,060 \\
\hline
\end{tabular}

* Se determinó utilizando un hidrómetro de campo que mide la relación entre el peso en aire y el peso en agua de una muestra de $3.166 \mathrm{~kg}$ de tubérculos. 
ALCANCES TECNOLÓGICOS, AÑO 4, NÚMERO 1

CUADRO 6. Rendimiento y características de tubérculo de 21 clones mexicanos y del C.I.P. 2002.

\begin{tabular}{ccccccc}
\hline CLON & $\begin{array}{c}\text { RENDIMIENTO } \\
(\mathbf{t} / \mathbf{h})\end{array}$ & $\begin{array}{c}\text { GRAVEDAD } \\
\text { ESPECIFICA }\end{array}$ & $\begin{array}{c}\text { Color } \\
\text { Piel }\end{array}$ & $\begin{array}{c}\text { CARTER } \\
\text { Pulpa }\end{array}$ & Forma & Profundidad \\
de ojos
\end{tabular}

\section{Año 2003}

Los resultados resumidos de estos ensayos se presentan en los Cuadros 7, 8 y 9 donde se muestran las características de rendimiento y calidad de tubérculo (físicas, químicas y sensoriales). Las variedades NOSARA (391683.80), INTAFRIT (387146.47) Y MALEKE (387096.11) de nuevo presentaron las características mencionadas para el año 2002 (adecuadas de alto rendimiento y buenas cualidades para su consumo fresco, así como para uso industrial (hojuelas) en el caso de INTAFRIT. Estos resultados reflejan comportamientos similares del cultivo cuando se evaluaron otras variedades bajo las condiciones de Costa Rica como lo reportan Avilés y Bolaños 1999; Bonilla (1998); Bonilla (2001a); y presenta un patrón similar al reportado por otros autores bajo condiciones diferentes en otros países (Harrison 1992; Moreno 2000). 
Bonilla: Nosara, Maleke e Intafrit nuenas variedades de papa (Solanum tuberosum) para Costa Rica.

CUADRO 7. Rendimiento y características de tubérculo de 10 clones y variedades de papa en ensayo de rendimiento. Año 2003.

\begin{tabular}{ccccc}
\hline $\begin{array}{c}\text { VARIEDAD/ } \\
\text { CLON }\end{array}$ & $\begin{array}{c}\text { RENDIMIENTO } \\
\text { COMERCIAL } \\
\mathbf{( k g )}\end{array}$ & $\begin{array}{c}\text { RENDIMIENTO } \\
\text { NO COMERCIAL } \\
\mathbf{( k g )}\end{array}$ & $\begin{array}{c}\text { GRAVEDAD } \\
\text { ESPECIFICA }\end{array}$ & $\begin{array}{c}\text { PORCENTAJE } \\
\text { SÓLIDOS }\end{array}$ \\
\hline Floresta & 11,8 & 3,9 & 1,064 & 17,0 \\
387146.47 & 13,6 & 5,0 & 1,068 & 21,8 \\
387096.11 & 38,4 & 4,6 & 1,063 & 16,6 \\
575049 & 14,1 & 8,5 & 1,062 & 16,4 \\
Idiap 92 & 7,9 & 4,0 & 1,060 & 16,1 \\
386042.3 & 12,0 & 9,1 & 1,069 & 17,9 \\
384321.15 & 14,0 & 9,0 & 1,067 & 17,4 \\
Idiafrit & 3,1 & 1,1 & 1,066 & 17,2 \\
391683.80 & 9,5 & 4,1 & 1,077 & 18,4 \\
Granola & 9,3 & 6,9 & 1,062 & 16,4 \\
\hline
\end{tabular}

CUADRO 8. Ensayo preliminar de características organolépticas de la variedad Floresta y tres clones promisorios. Año 2003.

\begin{tabular}{ccccccccc}
\hline Nombre & Área & $\begin{array}{c}\text { Rendimiento } \\
\text { (kilogramos) }\end{array}$ & Verdes & Deformes Pequeños & Reventados & Cortes & Cortes \\
\hline $\begin{array}{c}\text { Floresta } \\
\text { Clon }\end{array}$ & $0,75^{\star} 20$ & 50,0 & 3,2 & 1,7 & 6,1 & 1,4 & 2,2 & 2,2 \\
387146.47 & $0,75^{\star} 20$ & 54,0 & 2,7 & 2,0 & 9,6 & 10,3 & 1,8 & 1,8 \\
Clon & $0,75^{\star} 20$ & 37,0 & 2,5 & 4,6 & 4,2 & 1,0 & 1,0 & 1,0 \\
391683.80 & & & & & & & & \\
Idiafrit & $0,75^{\star} 80$ & 43,5 & 1,1 & 1,3 & 13,7 & 0,0 & 0,6 & 0,6 \\
Clon & $0,75^{\star} 10$ & 44,0 & 1,2 & 0,3 & 3,8 & 0,0 & 0,8 & 0,8 \\
387096.11 & & & & & & & & \\
\hline
\end{tabular}

CUADRO 9. Ensayo preliminar de características químicas de la variedad Floresta y tres clones promisorios. Año 2003.

\begin{tabular}{ccccc}
\hline $\begin{array}{c}\text { Calidad } \\
\text { Variedad }\end{array}$ & Lectura & \% $\mathbf{H}_{\mathbf{2}} \mathbf{0}$ & \% MS & \% Almidón \\
Floresta & 1,062 & 83,58 & 16,42 & 10,24 \\
Clon & 1,069 & 82,11 & 17,89 & 11,63 \\
387028.10 & & & & \\
Clon & 1,065 & 82,95 & 17,05 & 10,84 \\
387146.47 & & & & 11,83 \\
Clon & 1,070 & 81,90 & 18,10 & 11,83 \\
387096.11 & & & & \\
Clon & 1,070 & 81,90 & 18,10 & \\
391683.80 & & & & \\
\hline
\end{tabular}

Fuente: Ing. Marco Vinicio Castro, Ing. Daniel Saborío.Laboratorio poscosecha CIA-UCR. 


\section{CONCLUSIONES}

Los clones denominados 391683.80 (NOSARA), 387096.11(MALEKE) y 387146.47 (INTAFRIT) presentaron características de rendimiento, adaptación y calidad de producto adecuadas para ser considerados como nuevas variedades de papa. Durante el proceso de evaluación desde 1995 a 2003 mostraron características de alto rendimiento superior a $35 \mathrm{t} / \mathrm{ha}$, tolerancia a tizón tardío y buenas características para consumo fresco, en el caso de NOSARA y MALEKE, así como para hojuelas en el caso de INTAFRIT con valores superiores a $20 \%$ de materia seca.

\section{LITERATURA CITADA}

Avilés, J.; Bolaños, A. 1999.Evaluación de clones avanzados de papa (Solanum tuberosum) en dos localidades de la zona norte de Cartago. En: Memoria IX Congreso Nacional Agronómico y de Recursos Naturales Volumen II Manejo de Cultivos. p 286.

Beukema, H.P.; Van Der Zaag, D.E. 1990. Introduction to potato production. Ed. Pudoc, Wageningen, The Netherlands. 208p.

Bonilla, N. 1998. Evaluación de clones avanzados de papa con resistencia al Tizón Tardío (Phytophthora infestans L. Moench) y calidad industrial. Revista Investigación Agrícola. Costa Rica 7(1-2): 57-65.

------. 2001. Evaluación de clones promisorios de papa con resistencia a tizón tardío y calidad de tubérculo. En: Memorias de la XLVII Reunión del PCCMCA del 2001. Costa Rica. 60 p.

2001. Validación de clones de papa en diferentes zonas paperas de Costa Rica. En: Memorias de la XLVII Reunión del PCCMCA del 2001. Costa Rica. 60 p.

CNP (CONSEJO NACIONAL DE LA PRODUCCION, C.R.). 2000. Frutas y vegetales: base de datos en línea. San José, Costa Rica. Consultado el 10 de diciembre del 2000.

Disponible en

www.mercanet.cnp.go.cr/SIM/frutasyvegetales.

2000.F.A.O. (ORGANIZACIÓN MUNDIAL DE LAS NACIONES UNIDAS PARA LA AGRICULTURA Y LA ALIMENTACION)
1994. Anuario de Producción. Organización de las Naciones Unidas para la Agricultura y Alimentación. 47: 89-90.

Harrison, J.G. 1992. Effects of the aerial environment on late blight of potato foliagea review. Plant Pathology E.U. 41: 384-416.

Henflig, J.W. 1987. El tizón tardío de la papa (Phytophthora infestans). Centro Internacional de la Papa (C.I.P.). Lima, Perú. Boletín de Información Técnica 4. 25 p.

Hooker, W.J. 1981. Compendium of potato diseases. Editado por The American Phytopathology Society. E.U. 125 p.

Horton, D. 1992. La papa. Producción, Comercialización y Programas. Centro Internacional de la Papa (C.I.P.) Editorial Hemisferio Sur. Lima, Perú, Montevideo, Uruguay. $260 \mathrm{p}$.

Landeo, J.; Gastelo, M; Parraga, A.; Díaz, L. 2000. Progreso en el desarrollo de clones avanzados de papa con altos niveles de resistencia horizontal al tizón tardío (Phytophthora infestans Mont. de Bary) en la población B. Memoria Congreso ALAP 2000. Cuba. p 101.

Mendoza, A; Landeo, J; Gastelo, M.; González, A.; Morales, A.; Dominguez, F; Gayozzo, M. 2000. Chata Roja-Unheval, Nueva variedad de papa con resistencia duradera a la rancha (Phytophthora infestans Mont de Bary) para el Perú. p 99.

Moreno, J. 2000. Selección de clones de papa para la industria en Colombia. En Memoria Congreso ALAP 2000. Cuba. p 118.

Rodríguez, C. 1988. Propuesta para modificar la cuantificación del daño de polillas en tubérculos de papa. Revista Investigaciones Agrícolas. Costa Rica. 2(2): 39-41.

Umareus, V.; Umareus, M. 1994. Inheritance of resistance to late blight. En: Potato Genetics. [Editado por J.E. Bradshaw; G.R. Mackay] CAB. International. Wallingford, Oxford. U.K. p:365-402.

Vázquez, V. 1988. Mejoramiento Genético de la Papa. AMARV Editores. Lima, Perú. 208 p. 